\title{
Vestibular Signals in Macaque Extrastriate Visual Cortex Are Functionally Appropriate for Heading Perception
}

\author{
Sheng Liu and Dora E. Angelaki \\ Department of Neurobiology, Washington University School of Medicine, St. Louis, Missouri 63110
}

Visual and vestibular signals converge onto the dorsal medial superior temporal area (MSTd) of the macaque extrastriate visual cortex, which is thought to be involved in multisensory heading perception for spatial navigation. Peripheral otolith information, however, is ambiguous and cannot distinguish linear accelerations experienced during self-motion from those resulting from changes in spatial orientation relative to gravity. Here we show that, unlike peripheral vestibular sensors but similar to lobules 9 and 10 of the cerebellar vermis (nodulus and uvula), MSTd neurons respond selectively to heading and not to changes in orientation relative to gravity. In support of a role in heading perception, MSTd vestibular responses are also dominated by velocity-like temporal dynamics, which might optimize sensory integration with visual motion information. Unlike the cerebellar vermis, however, MSTd neurons also carry a spatial orientation-independent rotation signal from the semicircular canals, which could be useful in compensating for the effects of head rotation on the processing of optic flow. These findings show that vestibular signals in MSTd are appropriately processed to support a functional role in multisensory heading perception.

\section{Introduction}

How we orient and move in the world is encoded by sensory information from the visual and vestibular systems. The dorsal medial superior temporal area (MSTd) of extrastriate visual cortex is important for the processing of optic flow, i.e., the retinal flow patterns experienced during navigation (Gibson, 1950; Warren and Hannon, 1990; Warren, 2003). MSTd neurons have large receptive fields and respond to complex optic flow patterns (Tanaka et al., 1986; Duffy and Wurtz, 1991, 1997; Heuer and Britten, 2004; Logan and Duffy, 2006). In addition, microstimulation of MSTd biases heading judgments based on optic flow (Britten and van Wezel, 1998).

MSTd neurons are also tuned during actual self-motion (Duffy, 1998; Bremmer et al., 1999; Page and Duffy, 2003; Gu et al., 2006; Fetsch et al., 2007) and this selectivity is of vestibular origin (Takahashi et al., 2007). Furthermore, MSTd responses are correlated with perceptual decisions in a heading discrimination task based solely on vestibular cues (Gu et al., 2007). These findings are further corroborated by the fact that multimodal MSTd neurons with congruent visual and vestibular preferences show improved directional sensitivity under bimodal stimulation that parallels similar effects on behavior (Gu et al., 2008). Collectively, these results

\footnotetext{
Received April 2, 2009; revised May 23, 2009; accepted June 3, 2009.

Experiments were supported by National Institutes of Health Grants EY017866 and EY019087. We thank Tatyana Yakusheva, Yong Gu, David Dickman, and Greg DeAngelis for collegial contributions to this work. The content is solely the responsibility of the authors and does not necessarily represent the official views of the National Eye Institute or the National Institutes of Health.

Correspondence should be addressed to Dr. Dora E. Angelaki, Department of Neurobiology, Box 8108 , Washington University School of Medicine, 660 South Euclid Avenue, St. Louis M0 63110. E-mail: angelaki@cabernet.wustl.edu.

DOI:10.1523/JNEUROSCI.1607-09.2009

Copyright $\odot 2009$ Society for Neuroscience $\quad$ 0270-6474/09/298936-10\$15.00/0
}

suggest that vestibular signals in MSTd could be functionally relevant for sensory integration for heading perception.

But are vestibular signals in MSTd indeed appropriate for such a role in multisensory perception? First, for cross-modal integration, vestibular signals must be temporally matched to visual signals (Zupan et al., 2002). But early otolith signals encode acceleration (Fernández and Goldberg, 1976a; Si et al., 1997), whereas visual motion responses are typically velocity-like (Rodman and Albright, 1987; Lisberger and Movshon, 1999). Although the population peristimulus time histogram (PSTH) during heading along the preferred direction appears to at least qualitatively follow a velocity-like waveform (Gu et al., 2006), quantitative characterization of the dynamics of vestibular responses in MSTd is missing. Second, these early otolith signals suffer from a sensory ambiguity: They encode net linear acceleration and cannot distinguish those accelerations resulting from self-motion from changing spatial orientation relative to gravity (e.g., during tilt) (Angelaki et al., 2004; Dickman et al., 1991; Fernández and Goldberg, 1976a,b). Perceptually this ambiguity rarely constitutes a problem even in darkness, except for very prolonged periods of acceleration (i.e., at very low frequencies (Merfeld et al., 1999, 2005). This occurs because the sensory ambiguity in the vestibular periphery can be resolved centrally by combining signals from both vestibular sensors, the otolith organs and the semicircular canals (Angelaki et al., 1999; Merfeld and Zupan, 2002; Zupan et al., 2002; Green and Angelaki, 2003, 2004; Green et al., 2005; Shaikh et al., 2005b). Indeed, Yakusheva et al. $(2007,2008)$ have described such optimal canal/otolith convergence in Purkinje cells of the nodulus and uvula of the cerebellar vermis.

If vestibular responses in MSTd are indeed appropriate for heading perception, they should modulate selectively during selfmotion and not in response to changes in spatial orientation 
relative to gravity. Here we show that vestibular signals in MSTd are indeed temporally and functionally appropriate for such a role in heading perception.

\section{Materials and Methods}

Subjects and surgery. Three rhesus monkeys (Macaca mulatta, 3.5-6 kg) were chronically implanted with an eye coil, head-restraining ring, and a plastic guide tube platform for single unit recordings (see Meng et al., 2005; Gu et al., 2006 for details). All surgical procedures were performed in accordance to institutional and NIH guidelines.

Electrophysiological recordings. For these experiments, animals were seated in a primate chair that was secured inside a vestibular turntable consisting of a three-axis rotator on top of a linear sled (Acutronics Inc.). The system could deliver yaw, pitch, or roll rotation and translation along any direction in the horizontal plane (but not vertical translations, as in $\mathrm{Gu}$ et al., 2006; Takahashi et al., 2007). Animals were placed such that, when upright, their horizontal stereotaxic plane was aligned with the earth-horizontal and all three rotational axes (yaw, pitch, and roll) were aligned with the center of the head.

We recorded extracellular activity of single neurons in area MSTd using epoxy-coated tungsten microelectrodes (FHC; 1-2 M $\Omega$ ). Electrodes were inserted into 26-gauge transdural guide tubes and advanced by a remote-controlled microdrive (FHC). Neural activities were amplified, filtered $(300-6 \mathrm{kHz})$ and passed through a dual time-amplitude window discriminator (BAK Electronics). Note that, although horizontal and vertical eye movements were recorded as part of these experiments, they are not further analyzed here. Details about the three-dimensional eye movements evoked during the same experimental protocols as those used in the present experiments have been presented by Angelaki et al. (1999).

The vestibular-responsive region of area MSTd was identified using a combination of anatomical and electrophysiological criteria, as described in detail previously (Gu et al., 2006). Anatomical criteria were based both on stereotaxic coordinates and magnetic resonance imaging (MRI). Physiological criteria were as follows: (1) MSTd was usually the first gray matter modulating to flashing visual stimuli; (2) MSTd neurons had large receptive fields $(\mathrm{RF})$ that often included the contralateral visual field but could also extend into the ipsilateral visual field; (3) Finally, our penetrations in MSTd were also guided by the eccentricity of receptive fields in underlying area MT (Gu et al., 2006).

Experimental protocols. The microelectrode was advanced into area MSTd while the monkey performed a simple fixation task and the neuron's RF was mapped by moving a patch of drifting random dots around the visual field on a custom graphical interface (see Gu et al., 2006 for details). Then the monitor was turned off and the following protocols were all run in total darkness. Note that the sensitivity of MSTd neurons does not change significantly during self-motion in complete darkness versus fixation of a real or imaginary target ( $\mathrm{Gu}$ et al., 2006, 2007; Takahashi et al., 2007). The present experiments were all performed in total darkness and care was taken not to have any light leak from around the door of the laboratory. We chose to perform these experiments in total darkness and without any behavioral control for two main reasons. First, we wanted to exclude any contribution of retinal slip or attempt to fixate and suppress the vestibulo-ocular reflex. Second, we wanted to make direct comparisons with responses in thalamus, cerebellum and brainstem, where data were collected while the animals were allowed to make eye movements freely. Finally, note that, although MSTd neurons have robust responses to pursuit (Bremmer et al., 1997; Upadhyay et al., 2000; Page and Duffy, 2003; Ilg, 2008), they do not seem to systematically change their firing rate during the vestibulo-ocular reflex in darkness ( $\mathrm{Gu}$ et al., 2007).

To manipulate translational (inertial) and net (gravitoinertial) linear acceleration (Angelaki et al., 2004; Meng et al., 2007; Yakusheva et al., 2007), four stimuli were delivered: translation only, tilt only or combined translation and tilt ("tilt-translation" and "tilt+translation"). The tilt stimulus consisted of a $0.5 \mathrm{~Hz}$ sinusoidal rotation from an upright position with peak amplitude of $\pm 11.5^{\circ}$. This stimulus causes reorientation of the head relative to gravity, such that otolith afferents are stimulated by a $\pm 0.2 \mathrm{G}$ linear acceleration component in the head-horizontal plane. The amplitude of translation was then adjusted to match that induced by the head tilt $( \pm 0.2 \mathrm{G}$, resulting in a displacement of $\pm 20 \mathrm{~cm}$ ). During combined tilt and translation, the translational and gravitational accelerations combine in either an additive or subtractive manner, depending on the relative phase of the two stimuli. As a result, the net gravitoinertial acceleration in the horizontal plane either doubled (tilt + translation) or was nearly zero (tilt-translation), even though the actual translation remained the same. Each cell was tested at two orientations, $\theta=0^{\circ}$ and $\theta=$ $90^{\circ}$, corresponding to lateral motion/roll tilt and forward/backward motion/pitch tilt, respectively. Whenever single cell isolation was maintained, lateral and forward/backward translations were also delivered at different frequencies: $0.3 \mathrm{~Hz}( \pm 0.1 \mathrm{G}), 1 \mathrm{~Hz}( \pm 0.2 \mathrm{G})$, and $2 \mathrm{~Hz}( \pm 0.3$ $\mathrm{G})$.

In addition to pitch and roll tilts (which activate both otolith and semicircular canal afferents), neurons were also tested during rotations $\left(0.5 \mathrm{~Hz}, \pm 10^{\circ}\right)$ about an earth-vertical axis (EVR). Such rotations do not change head orientation relative to gravity, thus they activate exclusively semicircular canal (but not otolith) afferents. First, yaw (left-right) rotation was delivered with the animals seated upright. Next, to test vertical canal activation during EVR, the same motion was also delivered with the animal statically tilted: pitched $45^{\circ}$ nose-up/down (stimulating a plane half-way between yaw and roll) and/or rolled $45^{\circ}$ right/left ear-down (stimulating a plane half-way between yaw and pitch).

These data, collected during EVR (thus activating only the semicircular canals), were then used to compare with pitch/roll tilt responses (which activate both otolith organs and semicircular canals) and quantitatively test the hypothesis that rotation responses in MSTd do not depend on spatial orientation relative to gravity. These comparisons represent the best way to test the canal- versus otolith-driven origin of the MSTd tilt responses (see Results): if earth-horizontal (tilt) and earthvertical axis responses are identical, then they are gravity-independent and likely originate from the semicircular canals. On the other hand, if they are not identical, then we conclude that otolith-driven signals also contribute to the pitch/roll tilt modulation of MSTd cells. Note that we have used this experimental protocol previously in vestibular nuclei neurons: we found no correlation between the two, allowing us to conclude that some of the tilt responses arise from activation of the otolith organs (Dickman and Angelaki, 2002).

It is important to emphasize that this comparison could not be done by measuring MSTd activity during static tilt; the reason is that otolithdriven central responses are often strongly frequency-dependent (see Fig. 6) (see also Dickman and Angelaki, 2002; Yakusheva et al., 2008; Shaikh et al., 2005a); what happens under static tilt conditions and at $0.5 \mathrm{~Hz}$ can be quite different. In particular, if we found no static tilt sensitivity, it would have been incorrect to conclude that there is no otolith-driven tilt response at $0.5 \mathrm{~Hz}$ (since we cannot eliminate high-pass tilt dynamics for these neurons). Similarly, if we had found static tilt sensitivity, we could not have concluded that there is an otolith-driven contribution to $0.5 \mathrm{~Hz}$ tilt responses (since we cannot eliminate low-pass tilt dynamics). In fact, the simple spike responses of nodulus/uvula Purkinje cells modulate strongly with static tilt, but not during $0.5 \mathrm{~Hz}$ tilt, and this difference is striking (Yakusheva et al., 2007, 2008).

Data analyses. Permutation analysis was used to determine whether cells modulated significantly to each sinusoidal stimulus, as follows. Firing rates were first binned ( 40 bins per cycle) and a Fourier ratio (FR) was defined as the fundamental frequency over the maximum of the first 20 harmonics. Subsequently, the 40 response bins were shuffled randomly, thus destroying the systematic modulation in the data but maintaining the inherent variability of the responses. An FR was then computed from those randomly permuted histograms, and the randomization process was repeated 1000 times. If the FR for the original data exceeded that for $99 \%$ of the permuted data sets, we considered the temporal modulation to be statistically significant $(p<0.01)$.

Sinusoidal responses were further quantified using instantaneous firing rate (IFR) (computed as the inverse of interspike interval). First, IFRs from multiple cycles were folded into a single cycle by overlaying neural responses. Subsequently, amplitude and phase were determined by fitting a sine function (clipped off at zero response) to both response and 
stimulus using a nonlinear least-squares minimization algorithm (Levenberg-Marquardt). Response amplitude refers to half the peak-totrough modulation. For rotational stimuli, neural response gain was computed as the ratio of response amplitude over peak head velocity (in units of spikes/s per \%). For translational stimuli, neural gain was calculated as response amplitude divided by either peak acceleration ("acceleration" gains, in units of spikes/s per G; $\mathrm{G}=981 \mathrm{~cm} / \mathrm{s}^{2}$ ) or by peak velocity ("velocity" gains, in units of spikes/s per $\mathrm{cm} / \mathrm{s}$ ). Phase was expressed as the difference between peak response and peak velocity (rotation and tilt) or acceleration (translation).

The spatial tuning in the horizontal plane for translation and sagittal and frontal planes for rotation were quantified using a spatiotemporal cosine-like tuning model (Angelaki, 1991, 1992; Schor and Angelaki, 1992). In particular, the model has four parameters; three parameters characterize the properties of the cell's preferred stimulus: i.e., preferred direction, as well as response gain and phase for stimulation along the preferred direction. However, unlike the traditional cosine-tuning in which these three parameters are sufficient to characterize responses along any other direction, the spatiotemporal model has a fourth parameter, the response gain along a second response direction (the latter is always spatially and temporally orthogonal to the preferred direction). This fourth parameter is always assumed to be zero for traditional cosine-tuning. Note that the spatiotemporal model is more general than the traditional cosine-tuning model; whereas the latter assumes zero response for perpendicular directions, the spatiotemporal model allows for nonzero response along the axis perpendicular to the preferred direction. The larger the magnitude of this perpendicular response relative to the preferred response, the larger is the departure from the traditional cosine-tuning. In general, spatiotemporal tuning allows temporal dynamics and spatial properties to be intermingled, such that more than one temporal parameter (e.g., velocity and acceleration) can be simultaneously coded along different spatial directions. The spatiotemporal model was shown to characterize best the translation tuning of brainstem and cerebellar vestibular neurons (Bush et al., 1993; Angelaki and Dickman, 2000; Shaikh et al., 2005a; Chen-Huang and Peterson, 2006; Yakushin et al., 2006).

To determine whether cell responses correlated best with translation or net acceleration, linear regression analysis was used to simultaneously fit cumulative cycles of cell modulation during each of the translation, tilt and combined stimuli using "net acceleration" and "translation"-coding models (for details, see Angelaki et al., 2004; Green et al., 2005). To determine how well each of the two models fitted the data, we computed partial correlation coefficients, which were normalized using Fisher's $r$-to- $z$ transform.

\section{Results}

More than half of the recorded MSTd neurons (92 of 175, 53\%) had a significant response modulation (permutation test, $p<$ 0.01 ; see Materials and Methods) during motion in darkness (Table 1). These motions included yaw (left-right rotation), pitch (nose up-down rotation) and roll (left-right ear-down rotation), as well as lateral and forward/backward translation. Vestibular neurons included approximately equal percentages of translation- and rotation-responding cells (42\% and 39\%, respectively; see Table 1). Given that vertical motion-preferring neurons were not identified here, the percentage of translationresponding neurons in total darkness reported here $(42 \%)$ is slightly lower than the percentage of MSTd neurons (54-64\%) tuned to three-dimensional (3D) translation ( $\mathrm{Gu}$ et al., 2006, 2007; Takahashi et al., 2007). Similarly, the percentage of rotation-responding neurons reported here (39\%) is similar to those tuned to $3 \mathrm{D}$ rotation in darkness [note that during fixation the percentage of responding cells is higher because of a residual rotational vestibulo-ocular reflex causing retinal slip and thus evoking visual responses in many MSTd neurons during fixation (Takahashi et al., 2007; Chowdhury et al., 2008)]. The majority $(28 \%, 49$ of 175$)$ of cells were "convergent," i.e., they responded during both translation and rotation. Nonconvergent neurons were less frequent; 14\% (24 of 175) of MSTd cells were exclusively sensitive to translation and 11\% (19 of 175) modulated exclusively during rotation.

The spatial tuning in the horizontal plane for translation and sagittal and frontal planes for rotation were quantified using a spatiotemporal cosine-like tuning model (Angelaki, 1991, 1992; Schor and Angelaki, 1992) (see Materials and Methods). Preferred directions were broadly and uniformly distributed within the horizontal plane (uniformity test, $p=0.75$ ) (Fig. $1 A$ ), with gains averaging $269 \pm 22.7$ spikes/s/G at $0.5 \mathrm{~Hz}$ (range 39-1036). There was no difference in either gain or preferred direction for 


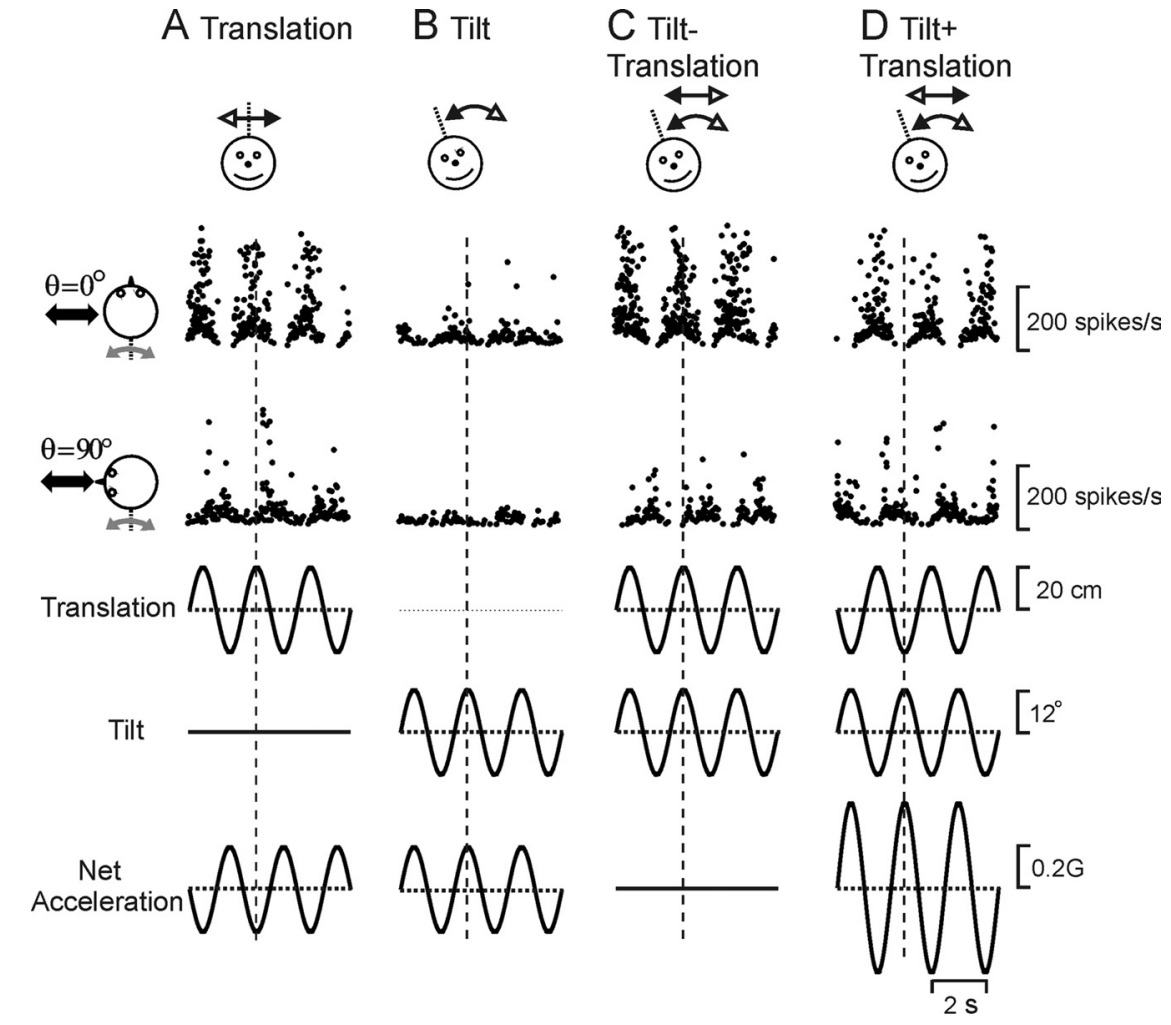

Figure 2. $\boldsymbol{A}-\boldsymbol{D}$, Example of MSTd neuron responses during combinations of tilt and translation: translation only $(\boldsymbol{A})$, tilt only $(\boldsymbol{B})$, and combined translation and tilt $(\boldsymbol{C}, \boldsymbol{D})$ (tilt-translation, where translational and gravitational accelerations cancelled each other and tilt + translation, where translational and gravitational accelerations added and net acceleration doubled). Data are shown along two stimulation axes (diagrams). Vertical dotted lines mark peak stimulus amplitude. This is an example of a convergent MSTd neuron that modulated significantly during roll, but not yaw and pitch rotation.

On the other hand, pitch/roll responses can also be otolith-driven, reflecting the sensitivity of the peripheral otolith sensors to gravitational acceleration. The latter, i.e., if pitch/roll responses are otolithdriven and caused by changes in orientation relative to gravity, would be inappropriate for driving heading perception; otherwise, every time we tilt our head it would be perceived as self-motion. Here we test whether the pitch/roll modulation of MSTd neurons arise from gravityresponsive, otolith-driven signals or spatial orientation-independent, canaldriven signals. First, we use translation and tilt, as well as combinations of translation and tilt, to show that MSTd responses correlate best with translation and not net acceleration. Next, we use rotations about different axes to show that rotational responses in MSTd are independent of head orientation relative to gravity.

\section{MSTd neurons correlate better with translation rather than with net linear acceleration}

To investigate whether MSTd neurons selectively encode true heading information or, like otolith afferents, they also modulate in response to gravitational acceleration, MSTd neurons were tested during translation, tilt and combination stimuli (Angelaki et al., 2004), as shown in the top schematics of Figure 2. Because peak tilt amplitude is such

convergent versus nonconvergent cells (Wilcoxon test, gain: $p=$ 0.11 ; preferred direction: $p=0.76$ ) (Fig. $1 A$, filled vs open symbols, respectively). Tuning ratios (i.e., the ratio of the minimum over maximum response gain) were unimodally distributed (modality test, $\left.p_{\text {uni }}=0.4\right)($ Fig. $1 B$ ). The majority of MSTd cells had tuning ratio close to zero, suggesting traditional cosine-tuning. A notable proportion (44\%, 32 of 73 ) of MSTd neurons, however, exhibited spatiotemporal properties, with response gains along a perpendicular direction that were larger than $20 \%$ of those along the preferred direction (tuning ratio $>0.2$ ). The distribution of neuronal phase was uniform (uniformity test, $p=0.3$ ) (Fig. 1C), as is typical of responses in other vestibular areas (Angelaki and Dickman, 2000; Shaikh et al., 2005a).

Lesion experiments have shown that the responses of MSTd neurons during self-motion in darkness are of labyrinthine origin (Gu et al., 2007; Takahashi et al., 2007). Specifically, translation responses arise from activation of the otolith organs and yaw rotation responses arise from activation of the semicircular canals. Pitch and roll modulation, however, can arise from activation of either the otolith organs or the semicircular canals. This occurs because otolith afferents are sensitive to net linear acceleration. Pitch and roll rotations (referred to here as "tilt") change the orientation of the head relative to gravity, thus providing an effective stimulus that activates both otolith organs and vertical semicircular canals. The origin of tilt modulation for MSTd neurons (Table 1) is crucial for their proposed role in heading perception. On the one hand, tilt responses might be of semicircular canal origin, thus reflecting a gravity-independent rotation signal that can be used for the processing of optic flow (see Discussion). that the horizontal linear acceleration caused by gravity is the same as that during translation (see Materials and Methods), when both translation and tilt are presented together, the net horizontal acceleration is either zeroed (tilt-translation) or doubled (0.4 G, tilt + translation, see Materials and Methods).

Representative responses from a typical MSTd cell during lateral translation/roll tilt $\left(\theta=0^{\circ}\right)$ and forward/backward translation/pitch tilt $\left(\theta=90^{\circ}\right)$ are shown in Figure 2. Although net acceleration was the same during translation and tilt (traces on bottom), most heading-sensitive MSTd neurons modulated more strongly during translation than during tilt (Fig. $2 A, B$, compare peak-to-trough sinusoidal modulation of firing rate). When translation and tilt are presented simultaneously, such that net horizontal linear acceleration is either zero (tilt-translation, Fig. 2C) or double (tilt + translation, Fig. 2D), MSTd neuron responses appear similar to those during translation.

Data from all heading-sensitive MSTd neurons are summarized in Figure 3. Across the population, responses during tilt are significantly attenuated compared with those during translation (Wilcoxon rank test, $p \ll 0.001$ ) (Fig. $3 A$ ). In addition, as expected from neurons that selectively encode translation and ignore changes in orientation relative to gravity (solid red lines), responses during tilt-translation and tilt + translation are similar to those during translation (Fig. 3, $B$ and $C$, respectively; Wilcoxon rank test, $p \gg 0.05$ ). Correlation slopes are not significantly different from unity (tilt-translation vs translation: 0.96 (95\% confidence interval: $[0.84,1.1], r=0.88, p \ll 0.001)$ and tilt + translation vs translation: 0.93 (95\% confidence interval: $[0.81,1.06], r=0.88, p \ll 0.001)$. In contrast, for both combi- 
nation stimuli, responses are inconsistent with the predictions of net linear acceleration (Fig. $3 B, C$, dashed blue lines). This is most striking during tilt-translation, where net linear acceleration is zero; data points fall along the diagonal axis and not along the abscissa (Fig. 3B). Thus, during combined stimulation the tilt movement is ignored and the cells modulate exclusively to the translational component of the motion. In fact, the translation and tilt responses of MSTd neurons sum and subtract linearly to generate the responses to combination stimuli, tilt + translation and tilt-translation (Fig. 4). Briefly, for each cell, we computed the vectorial sum and difference between the translation and tilt response and compared the computed gain and phase with those measured during tilt +translation and tilt-translation, respectively. There was no significant difference between either gain or phase of actually measured and predicted tilt + translation and tilt-translation responses (Wilcoxon rank test, $p>0.05$ ). We conclude that the stimuli we used operate on the linear range of MSTd cells. Note that these observations are true for both nonconvergent and convergent cell types; i.e., those that only modulate in response to translation and those with significant modulation during both rotation and translation (Figs. 3 and 4 , open vs filled symbols).

The finding that heading responses in MSTd reflect true selfmotion sensitivity that is independent of gravity is further corroborated by examining response phase (Fig. $3 D-F$ ). Note that tilt + translation and tilt-translation stimuli differ in the relative phase of the translation and tilt (Fig. 2C,D, bottom traces marked translation and tilt) and that response phase here has been expressed relative to tilt (which is the same for both stimuli). Thus, if indeed the modulation of MSTd neurons reflects selective coding of translation rather than net acceleration, neuronal phase during tilt-translation and translation should be the same; thus, data should fall along the unity-slope, red line (Fig. $3 E$ ). But neuronal phase during tilt + translation should be opposite (i.e., different by $180^{\circ}$ ) to that during translation (Fig. $3 F$, solid red lines). In contrast, if neurons respond to net acceleration, tilt + translation phase should be the same as that during translation (Fig. 3F, dashed blue lines). Data are clustered around the predictions for coding translation and not net acceleration (Fig. $3 E, F)$.

To quantify these observations, multiple linear regression analysis was used to compute partial correlation coefficients of how well each neuron's response to translation, tilt, tilttranslation and tilt + translation could be predicted by net acceleration (Fig. 2, bottom traces) or translation-coding models (Fig. 2, third row from bottom). To simplify plotting and visual interpretation, Fisher's $r$-to- $z$ transform was used to normalize the variances of partial correlation coefficients (Angelaki et al., 2004). Figure 5 shows a scatter plot of the $z$-transformed partial correlation coefficients, where dotted lines mark the 0.01 level of significance. Most MSTd neurons $(84 \%, 57$ of 68$)$ fall in the upper-left quadrant, illustrating that


C Tilt + Translation

Figure 3. $\boldsymbol{A}-\boldsymbol{F}$, Summary of neural response amplitude and phase during tilt $(\boldsymbol{A}, \boldsymbol{D})$, tilt-translation $(\boldsymbol{B}, \boldsymbol{E})$, and tilt + translation

Phas
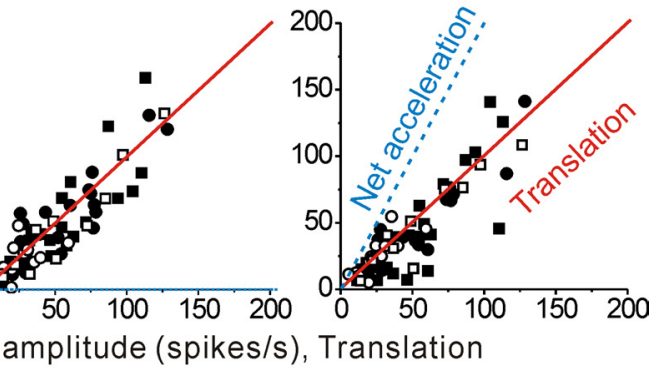

$\mathrm{F}$
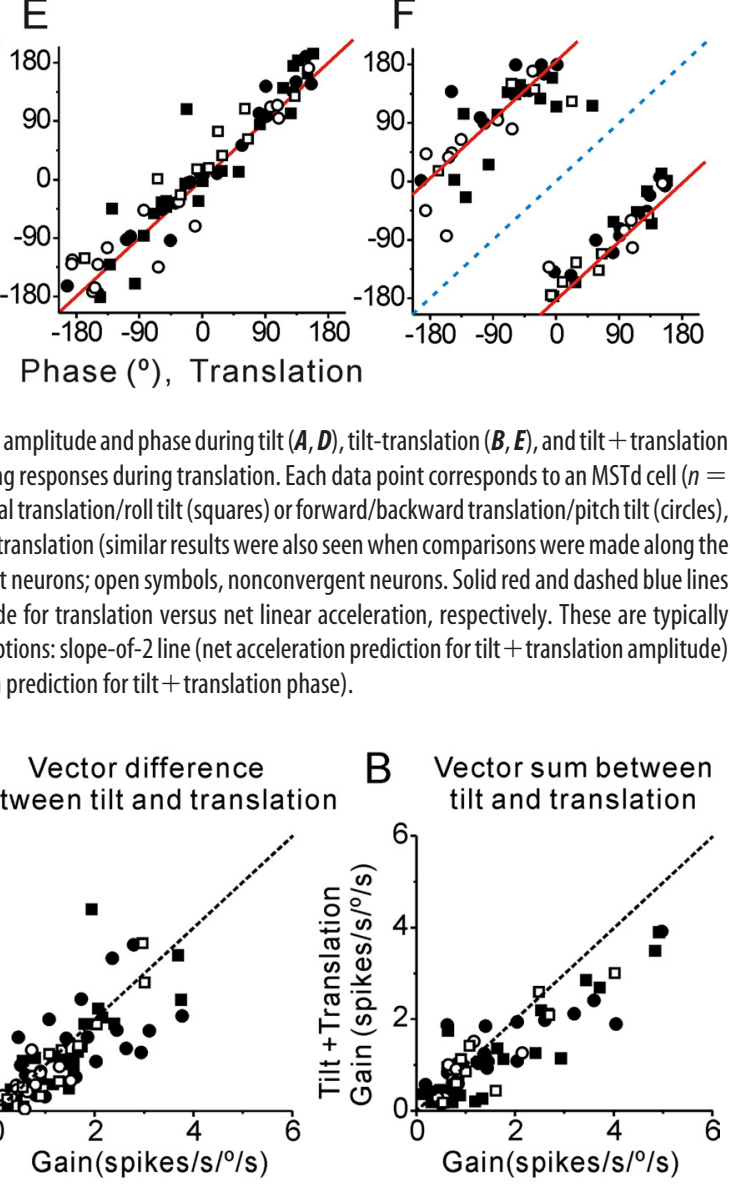


Figure 4. Test of response linearity. $\boldsymbol{A}, \boldsymbol{B}$, Comparison of tilt-translation $(\boldsymbol{A})$ and tilt + translation $(\boldsymbol{B})$ responses (ordinate) with the vector difference and sum (abscissa), respectively, of the neuronal responses during tilt and translation $(n=68)$. Note that these comparisons have been made to raw gain and phase measures along the axis of maximum translational response for each cell.

their firing rates are better correlated with coding of translation. Only $2.9 \%$ (2 of 68 ) are better correlated with net acceleration (Fig. 5, lower-right quadrant). This distribution of partial correlation coefficients is not different from that in the 


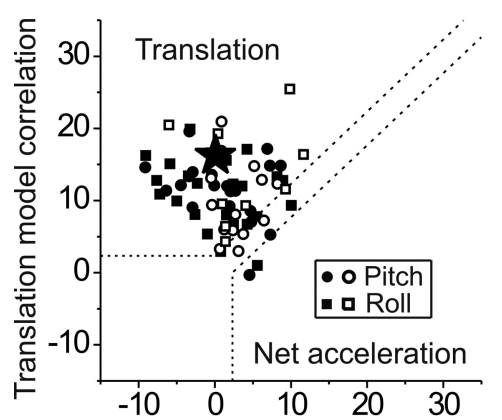

Net acceleration model correlation

Figure 5. Scatter plots of $z$-transformed partial correlation coefficients for fits of each cell responses with translation and net acceleration models. The superimposed dashed lines divide the plots into three regions: an upper/left area corresponding to responses significantly better fit by the translation-coding model; a lower/right area includes neurons which were significantly better fit by the net acceleration-coding model; and an in-between area in which responses would not be significantly better fit by either model at a $p=0.01$ significance level. As in Figure 3, each data point corresponds to a cell $(n=68)$, shown only for the best-responding direction of the cell: lateral translation/roll tilt (squares) or forward/backward translation/pitch tilt (circles). Filled symbols, Convergent neurons; open symbols, nonconvergent neurons. Asterisk marks the cell whose responses are shown in Figure 2.

and Fig. $3 A$ ), the analysis of combinations of tilt and translation in Figure 5 does not support the hypothesis that these responses are otolith-driven. As will be shown next, a complementary analysis focusing on rotation responses further demonstrates that MSTd neurons: (1) ignore the otolith activation during tilt; and (2) tilt (rotation) responses reflect gravity-independent signals, likely arising from the semicircular canals. But before we present that analysis, we first describe the frequency dynamics of heading responses of MSTd neurons. Note that, other than a qualitative description of the population PSTH along the preferred direction (Gu et al., 2006), MSTd response dynamics to translation have not been previously quantified.

\section{Response dynamics}

Translation at different frequencies was used to address whether heading responses are acceleration-like, similar to otolith afferents, or velocity-like, similar to visual responses to optic flow stimulation. We found that translation responses are largest at low frequencies, reaching $442 \pm 49$ spikes $/ \mathrm{s} / \mathrm{G}$ at $0.3 \mathrm{~Hz}$, but decreased with increasing frequency, as illustrated with a typical example in Figure $6 \mathrm{~A}$. Acceleration gains (i.e., ratio of peak response over peak acceleration) decrease with increasing frequency (Fig. 6B; ANCOVA, $p<0.001$, slope: -0.57). Response phase was independent of frequency (Fig. 6C; ANCOVA, $p>0.05$ ) [note that MSTd cells exhibit nonminimum phase characteristics, i.e., phase does not follow similar dependence on frequency as gain; this is typical of all central translation responses (Angelaki and Dickman, 2000; Dickman and Angelaki, 2002; Shaikh et al., 2005a; Yakusheva et al., 2008)]. The decreasing acceleration gain versus frequency plot implies that MSTd neurons, unlike otolith afferents, do not encode acceleration (otherwise acceleration gains would be flat and independent of frequency). A negative unity slope (when plotted in a log-log manner as in Fig. 6B) would indicate that MSTd neurons encode linear velocity. Thus, the less than unity slope in Figure $6 B$ suggests that MSTd neurons encode combinations of velocity and acceleration. This is further shown in Figure $6 \mathrm{D}$, which plots mean ( \pm SEM) acceleration (filled black circles) and mean velocity gains (filled gray squares). As expected, acceleration gains decrease with frequency, but velocity gains increase with frequency (ANCOVA, $p<$ 0.001, slope: 0.43 ).

Figure 6. Response dynamics of MSTd neurons during translation. $\boldsymbol{A}$, Example of translation responses at different frequencies. $\boldsymbol{B}, \boldsymbol{C}$, Neuronal gain and phase (expressed relative to linear acceleration and computed along the preferred direction; see Materials and Methods) plotted versus frequency. Thin gray lines show data from individual neurons ( $n=40)$. Thick black lines illustrate averages. $\boldsymbol{D}$, Average MSTd gains (filled symbols, solid lines) are compared with corresponding data from nodulus/uvula Purkinje cells (open symbols, dashed lines). Both acceleration (black symbols/line, left ordinate) and velocity (gray symbols/line, right ordinate) gains are shown. Note that neural response gains are computed along the preferred direction. Purkinje cell data are replotted with permission from Yakusheva et al., 2008 (copyright Society for Neuroscience).

nodulus/uvula (MANOVA, $p=0.1$ ) (Yakusheva et al., 2007), but differs from the medial vestibular nuclei (MANOVA, $p<$ 0.001) (Angelaki et al., 2004) and ventrolateral thalamus (MANOVA, $p<0.001$ ) (Meng et al., 2007), where data spanned the whole range and many neurons had net acceleration-like properties.

These results allow us to conclude that translation responses in MSTd encode true heading information and not net linear acceleration; that is, MSTd neurons are not sensitive to the component of otolith activation that results from changes in spatial orientation relative to gravity. Importantly, although several MSTd neurons modulate during pitch and roll tilt (see Table 1
The MSTd response dynamics differ from those in the vestibular nuclei (Angelaki and Dickman, 2000; Dickman and Angelaki, 2002) and fastigial nuclei (Shaikh et al., 2005a), where a mixture of increasing, decreasing and flat acceleration gains and strong phase dependence on frequency have been reported. However, both mean gain and its frequency dependence are identical to those of nodulus/uvula Purkinje cells (Yakusheva et al., 2008) (Fig. 6D, open symbols and dashed lines) (ANCOVA, main effect comparing MSTd and Purkinje cell data: $F_{(1,245)}=1.9, p=0.17$; interaction: $F_{(3,240)}=0.5, p=$ $0.7)$. Velocity-like responses have been suggested based on population PSTHs (Gu et al., 2006), but here we have quanti- 
fied that MSTd heading responses carry combinations of velocity and acceleration signals

\section{MSTd rotation responses are independent of spatial orientation relative to gravity}

Another way to test whether MSTd neurons indeed ignore the otolith activation during tilt is by showing that their modulation during rotation is independent of spatial orientation relative to gravity. The rationale is as follows. Rotation responses from the semicircular canals are independent of how the head is oriented relative to gravity. In contrast, rotation (i.e., tilt) responses of otolith afferents depend on how the head moves relative to gravity. Thus, our goal is to test whether rotational responses in MSTd depend on how the head is oriented relative to gravity. That is, for the same rotation plane relative to the head (i.e., pitch or roll), we could compare individual MSTd cell activity in response to tilt (i.e., earth-horizontal axis rotations that change head orientation relative to gravity) and EVR (i.e., earth-vertical axis rotations that do not change head orientation relative to gravity). If rotation modulation in MSTd arises exclusively from activation of the semicircular canals, we expect that tilt and EVR responses would be identical. Alternatively, if pitch and roll MSTd responses (Table 1) arise at least partially from otolith activation, tilt and EVR responses would be expected to differ (since the latter only activates semicircular canals, whereas the former incorporates both otolith and semicircular canal signals).

How MSTd cells respond during pitch and roll tilt has already been described (Table 1 and Fig. 3A). But how do we test for EVR pitch and roll responses? With the animal upright, this is impossible. To characterize EVR pitch/roll responses, macaques must be statically positioned $90^{\circ}$ ear-down (to test pitch) and supine or prone (to test roll; see schematics in Fig. $7 A$, top). In practice, $90^{\circ}$ repositioning is difficult; to avoid risk of losing cell isolation, we recorded MSTd neuron activities during EVR with the animals repositioned up to $\pm 45^{\circ}$ from upright, thus testing planes half-way between yaw and pitch/roll (Fig. $7 A$, top schematics). We then used both the yaw and yaw + roll or yaw + pitch EVR modulation to construct spatial tuning curves using the spatiotemporal cosine-like tuning model (see Materials and Methods) and computed the corresponding EVR pitch and roll modulation of the cell.

Responses from a typical MSTd cell during EVR stimulation with the animal positioned not only upright (yaw rotation), but also pitched $45^{\circ}$ nose-up/down (resulting in a combination of yaw + roll rotations) and rolled $45^{\circ}$ right/left ear-down (resulting in a combination of yaw + pitch rotations) are illustrated in Fig-

B
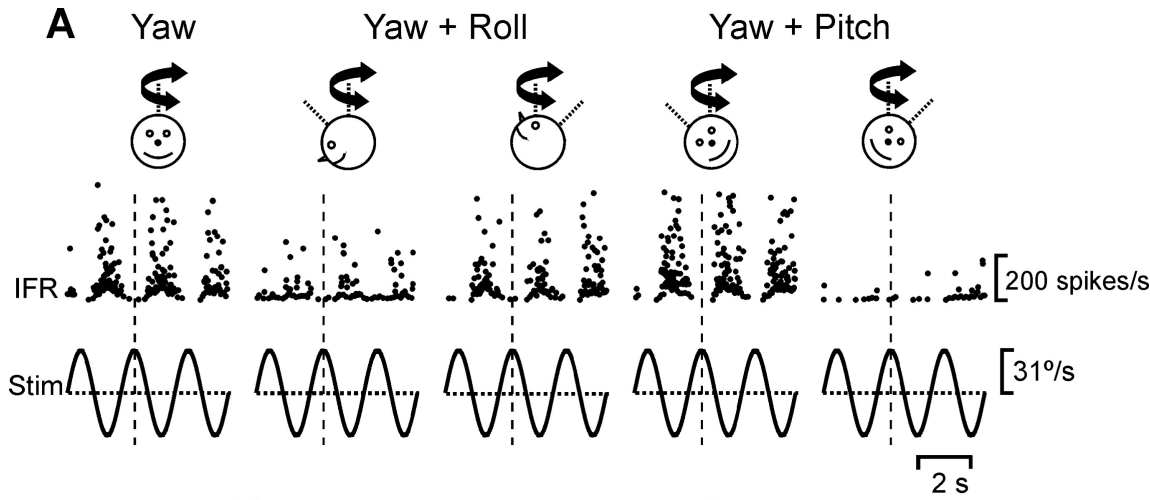

C

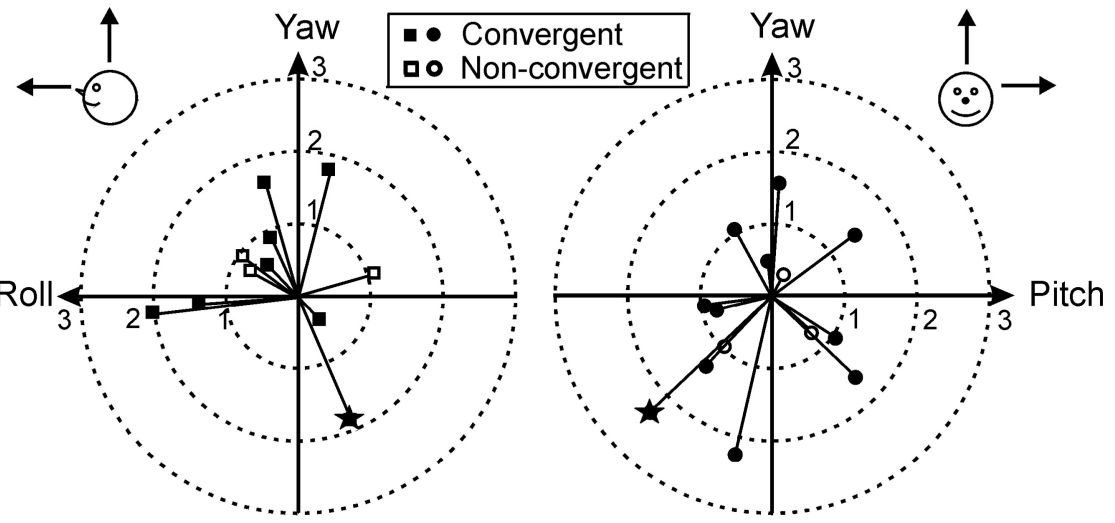

$E$

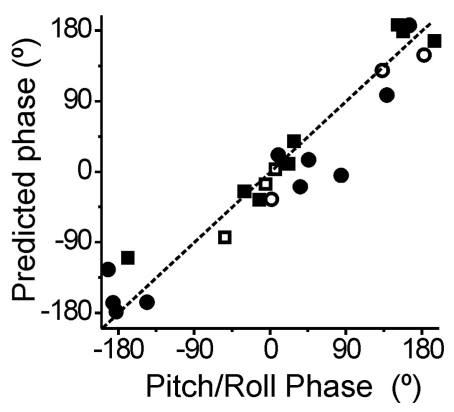

Figure 7. Summary of rotational preferred directions. $\boldsymbol{A}$, Instantaneous firing rate of an example MSTd cell during EVR with the monkey either upright (yaw) or statically tilted $\pm 45^{\circ}$, bringing the plane of rotation half-way between yaw and roll or pitch (see diagram drawings; note that the animal was tilted in the pitch plan to provide an EVR stimulus in a yaw + roll plane; and vice those in $\boldsymbol{A}$ ). Each data point corresponds to a cell, with distance from the origin reflecting its gain (in units of spikes $/ \mathrm{s} / \%$ ). Asterisk marks the cell whose responses are shown in $\boldsymbol{A} . \boldsymbol{D}, \boldsymbol{E}$, Comparison of the pitch (circles) and/or roll (squares) gains predicted from the EVR spatial tuning with those measured during pitch/roll tilt. Dotted lines illustrate unity-slope lines.

ure $7 A$. Figure $7, B$ and $C$, shows the distribution of preferred rotational directions in each of the sagittal and frontal planes, respectively. Each symbol in the plots corresponds to a convergent neuron (i.e., a cell modulating significantly during both rotation and translation; filled symbols) or nonconvergent neuron (i.e., a cell modulating only during rotation; open symbols), with the distance from the origin corresponding to its gain. From these plots (and corresponding tuning curves), we then calculated the predicted response to EVR pitch and roll rotations. Figure 7, D and $E$, compares the predicted EVR pitch/roll gain and phase with those measured during pitch/roll tilt for 14 MSTd cells. Both sets of parameters were not significantly different (Wilcoxon rank test, gain: $p=0.77$; phase: $p=0.82$ ). The fact that data fall along the diagonal suggests that tilt and EVR responses are not 
encoded differently by MSTd neurons. Given that the two stimuli differ only in terms of spatial orientation relative to gravity, we conclude that the rotational responses of MSTd cells reflect a (presumably canal-driven), spatial orientation-independent signal. Like the tilt/translation analysis (Fig. 5), this property of MSTd neurons also differs from brainstem responses: Unlike MSTd (Fig. 7), EVR and tilt responses in convergent vestibular nuclei neurons are not identical, reflecting the fact that a component of the tilt response is otolith-driven (Dickman and Angelaki, 2002).

\section{Discussion}

We have used traditional vestibular stimulation to characterize how neurons in an extrastriate visual cortical area, which is believed to be functionally linked to heading perception (Britten and van Wezel, 1998; Gu et al., 2007, 2008), respond during self-motion in darkness and how they compare with those in the brainstem and cerebellar cortex. Two response properties that are particularly relevant to multisensory heading perception were explored here. First, we show that MSTd neurons code combinations of heading velocity and acceleration. Responses closer to velocity make the vestibular modulation of MSTd neurons more similar and likely more compatible with the velocity-like responses to optic flow (Rodman and Albright, 1987; Lisberger and Movshon, 1999; Gu et al., 2006). Second, we show that MSTd vestibular responses are transformed from otolith afferent signals such that they are selective to the motions experienced during navigation (heading) and do not represent generic responses to net linear acceleration. In particular, we have shown that MSTd neurons do not respond to changes in spatial orientation relative to gravity, although they do carry an independent, presumably canal-driven, rotation signal (see below). Such selective coding of heading, over net linear acceleration, is appropriate for the proposed role of MSTd in visual/vestibular multisensory cue integration for self-motion perception.

This finding contrasts with a broader representation of both heading-specific and net linear acceleration signals in brainstem and thalamic nuclei (Angelaki et al., 2004; Meng et al., 2007). MSTd heading responses are instead similar to those of vermal Purkinje cells (Yakusheva et al., 2007). Interestingly, the two populations are also similar in other respects, including modulation amplitude and temporal properties (Fig. 4D). One difference between MSTd and cerebellar cortex responses is that the latter do not modulate during earth-vertical axis rotations (Yakusheva et al., 2007, 2008). In contrast, MSTd neurons do: unlike vermal Purkinje cells, a little less than half of MSTd neurons also carry a heading-independent, rotation signal (see also Takahashi et al., 2007). We have shown here that rotation signals in MSTd are independent of spatial orientation relative to gravity and thus likely reflect semicircular canal-driven (and not otolith-driven, tilt-related) signals.

Translation vestibular signals in MSTd are thought to be functionally linked to multisensory integration for heading perception (Britten and van Wezel, 1998, 2002; Gu et al., 2007, 2008). What, then, might be the function of rotation responses? One obvious role would be to disambiguate optic flow that is produced by self-translation from that produced by eye/head/body rotation (Royden et al., 1992; Banks et al., 1996; Crowell et al., 1998). Physiological studies have shown that MSTd neurons can signal heading from optic flow in the presence of pursuit eye movements (Bradley et al., 1996; Page and Duffy, 1999; Shenoy et al., 1999). By analogy, vestibular rotation signals in MSTd may be involved in compensating for the effects of head rotations on the processing of optic flow (see Takahashi et al., 2007, where this hypothesis was introduced). The different functional role of rotation and translation signals in MSTd is consistent with the fact that mainly translational components of optic flow are used for navigation; rotational optic flow is typically nulled by a compensatory VOR (Angelaki and Hess, 2005).

An important, and perhaps surprising, finding of the present study is the remarkable similarity between the heading properties of MSTd neurons and those of Purkinje cells in vermal lobules 9 and 10 (uvula and nodulus; Yakusheva et al., 2007, 2008). If MSTd and nodulus/uvula are somehow interconnected (and in later sections we postulate they are), this could only be through polysynaptic pathways. Let us first consider the afferents of the nodulus/uvula. Purkinje cells from the nodulus/uvula inhibit neurons in the vestibular and rostral fastigial nuclei (Shojaku et al., 1987; Wylie et al., 1994; Voogd et al., 1996; Fushiki and Barmack, 1997). Vestibular and cerebellar nuclei then project to the ventral lateral and ventral posterior lateral nuclei of the thalamus (Lang et al., 1979; Asanuma et al., 1983; Meng et al., 2001, 2007; Marlinski and McCrea, 2009), although it is presently unclear whether thalamus-projecting neurons are also nodulus/uvulatarget cells. The cortical projections of vestibular-responsive cells in the thalamus are also unknown, yet it is unlikely that their targets include MSTd, since thalamic inputs to MSTd appear limited to the inferior/medial pulvinar (Boussaoud et al., 1992; Kaas and Lyon, 2007), areas that do not modulate during vestibular stimulation (Meng and Angelaki, 2008).

Thus, most likely, vestibular signals reach MSTd after a minimum of four synapses, through corticocortical pathways, potentially involving the frontal eye fields (Ebata et al., 2004) and parieto-insular vestibular cortex (PIVC) (Guldin et al., 1992). Shorter-latency connectivity between MSTd and the cerebellum is more likely when considering the efferents to the nodulus/ uvula. MSTd has strong projections to the pretectum and pontine nuclei (Boussaoud et al., 1992; Distler et al., 2002), which give rise to both mossy and climbing fibers (the latter through the dorsal cap and ventrolateral outgrowth of the principal olive) (Voogd et al., 1996; Barmack, 2006). Alternatively to the possibility that responses in one area are driven (indirectly) by those in the other, it is equally likely that similar computations have been performed independently in different parts of the brain. At present, our findings cannot help distinguish among these alternatives.

Based on transneuronal tracing methods involving mainly the cerebellar hemispheres and dentate nucleus, Strick and colleagues (Middleton and Strick, 2001; Dum and Strick, 2003; Kelly and Strick, 2003) have shown that closed-loop circuits might represent a fundamental architectural feature of cerebrocerebellar interactions. Although not yet verified experimentally, the underlying assumption behind such closed-loop anatomical connections is similarity in physiological properties and underlying function. The similarity in response properties between MSTd and nodulus/uvula might represent a physiological signature of a yet-unidentified interconnectivity and linked function. Although this idea remains merely a hypothesis at present, the current findings provide strong motivation to search for such multisynaptic connectivity. We hypothesize that there is a functional link between MSTd (and perhaps other extrastriate cortical areas with a role in heading perception; e.g., VIP) (for review, see Britten, 2008) and nodulus/uvula, which could be mediated by closed-loop anatomical circuits, an emerging architecture of cerebro-cerebellar interactions (Strick et al., 2009).

Unlike the similarity in heading properties with the nodulus/ uvula, MSTd responses differ from those in the thalamus and brain- 
stem/cerebellar nuclei projecting to the thalamus (Angelaki and Dickman, 2000; Dickman and Angelaki, 2002; Musallam and Tomlinson, 2002; Shaikh et al., 2005a; Meng et al., 2007; for review, see Angelaki and Cullen, 2008). Neurons in these subcortical vestibular areas carry mixtures of translation and net acceleration signals and exhibit a wide variety of response dynamics with acceleration gains and phases that remain flat, increase or decrease as a function of frequency. Given that vestibular signals reach the cortex through vestibular, deep cerebellar nuclei and thalamus projections, such differences in response properties are surprising. Does the encoding of heading-related signals really change in these intermediate structures, or is it simply the case that the signals in MSTd and uvula/ nodulus are a subset of what is found in the vestibular/cerebellar/ thalamic nuclei? No data are currently available to distinguish among these alternatives.

The present findings support the postulated role of MSTd in multisensory heading perception. But, what is the functional role of the nodulus/uvula? Are these areas also involved in self-motion perception? The cerebellum has long been implicated in movement adaptation and internal models (Shidara et al., 1993; Wolpert et al., 1995; Glasauer, 2003; Green et al., 2007; Ghasia et al., 2008). In particular, the cerebellum is thought to construct "forward" models, whose function is to predict the consequences of the motor command on behavior, a signal critical to refining the motor command by computing an error between predicted and desired action. Although the concept of internal models has been particularly influential for motor control (Wolpert and Miall, 1996; Kawato and Wolpert, 1998; Kawato, 1999; Hwang and Shadmehr, 2005; Ito, 2005), it is also becoming increasingly popular for spatiotemporal sensory processing for multisensory perception (Merfeld et al., 1999; Angelaki et al., 2004; Zupan et al., 2004; Glasauer et al., 2007). The fact that the nodulus/uvula encodes true heading information, like cortical areas that presumably mediate perception (Gu et al., 2007, 2008), is consistent with the notion that the cerebellum maintains internal models of the sensory world and how it is encoded by the brain (i.e., an internal model of heading perception). Such internal model of sensory perception, similar to a forward model in motor control, can be fundamental in perceptual learning (Gilbert et al., 2001; Tsodyks and Gilbert, 2004) and construction/implementation of Bayesian priors (Jacobs, 1999; Knill and Pouget, 2004; Stocker and Simoncelli, 2006).

\section{References}

Angelaki DE (1991) Dynamic polarization vector of spatially tuned neurons. IEEE Trans Biomed Eng 38:1053-1060.

Angelaki DE (1992) Spatiotemporal convergence (STC) in otolith neurons. Biol Cybern 67:83-96.

Angelaki DE, Cullen KE (2008) Vestibular system: the many facets of a multimodal sense. Annu Rev Neurosci 31:125-150.

Angelaki DE, Dickman JD (2000) Spatiotemporal processing of linear acceleration: primary afferent and central vestibular neuron responses. J Neurophysiol 84:2113-2132.

Angelaki DE, Hess BJ (2005) Self-motion-induced eye movements: effects on visual acuity and navigation. Nat Rev Neurosci 6:966-976.

Angelaki DE, McHenry MQ, Dickman JD, Newlands SD, Hess BJ (1999) Computation of inertial motion: neural strategies to resolve ambiguous otolith information. J Neurosci 19:316-327.

Angelaki DE, Shaikh AG, Green AM, Dickman JD (2004) Neurons compute internal models of the physical laws of motion. Nature 430:560-564.

Asanuma C, Thach WT, Jones EG (1983) Distribution of cerebellar terminations and their relation to other afferent terminations in the ventral lateral thalamic region of the monkey. Brain Res 286:237-265.

Banks MS, Ehrlich SM, Backus BT, Crowell JA (1996) Estimating heading during real and simulated eye movements. Vision Res 36:431-443.

Barmack NH (2006) Inferior olive and oculomotor system. Prog Brain Res 151:269-291.
Boussaoud D, Desimone R, Ungerleider LG (1992) Subcortical connections of visual areas MST and FST in macaques. Vis Neurosci 9:291-302.

Bradley DC, Maxwell M, Andersen RA, Banks MS, Shenoy KV (1996) Mechanisms of heading perception in primate visual cortex. Science 273:1544-1547.

Bremmer F, Ilg UJ, Thiele A, Distler C, Hoffmann KP (1997) Eye position effects in monkey cortex. I. Visual and pursuit-related activity in extrastriate areas MT and MST. J Neurophysiol 77:944-961.

Bremmer F, Kubischik M, Pekel M, Lappe M, Hoffmann KP (1999) Linear vestibular self-motion signals in monkey medial superior temporal area. Ann N Y Acad Sci 871:272-281.

Britten KH (2008) Mechanisms of self-motion perception. Annu Rev Neurosci 31:389-410.

Britten KH, van Wezel RJ (1998) Electrical microstimulation of cortical area MST biases heading perception in monkeys. Nat Neurosci 1:59-63.

Britten KH, Van Wezel RJ (2002) Area MST and heading perception in macaque monkeys. Cereb Cortex 12:692-701.

Bush GA, Perachio AA, Angelaki DE (1993) Encoding of head acceleration in vestibular neurons. I. Spatiotemporal response properties to linear acceleration. J Neurophysiol 69:2039-2055.

Chen-Huang C, Peterson BW (2006) Three dimensional spatial-temporal convergence of otolith related signals in vestibular only neurons in squirrel monkeys. Exp Brain Res 168:410-426.

Chowdhury SA, Takahashi K, Deangelis GC, Angelaki DE (2008) Does area MT carry vestibular signals related to self-motion? Soc Neurosci Abstr 34:460.12.

Crowell JA, Banks MS, Shenoy KV, Andersen RA (1998) Visual self-motion perception during head turns. Nat Neurosci 1:732-737.

Dickman JD, Angelaki DE (2002) Vestibular convergence patterns in vestibular nuclei neurons of alert primates. J Neurophysiol 88:3518 -3533.

Dickman JD, Angelaki DE, Correia MJ (1991) Response properties of gerbil otolith afferents to small angle pitch and roll tilts. Brain Res 556:303-310.

Distler C, Mustari MJ, Hoffmann KP (2002) Cortical projections to the nucleus of the optic tract and dorsal terminal nucleus and to the dorsolateral pontine nucleus in macaques: a dual retrograde tracing study. J Comp Neurol 444:144-158.

Duffy CJ (1998) MST neurons respond to optic flow and translational movement. J Neurophysiol 80:1816-1827.

Duffy CJ, Wurtz RH (1991) Sensitivity of MST neurons to optic flow stimuli. I. A continuum of response selectivity to large-field stimuli. J Neurophysiol 65:1329-1345.

Duffy CJ, Wurtz RH (1997) Medial superior temporal area neurons respond to speed patterns in optic flow. J Neurosci 17:2839-2851.

Dum RP, Strick PL (2003) An unfolded map of the cerebellar dentate nucleus and its projections to the cerebral cortex. J Neurophysiol 89:634-639.

Ebata S, Sugiuchi Y, Izawa Y, Shinomiya K, Shinoda Y (2004) Vestibular projection to the periarcuate cortex in the monkey. Neurosci Res 49:55-68.

Fernández C, Goldberg JM (1976a) Physiology of peripheral neurons innervating otolith organs of the squirrel monkey. I. Response to static tilts and to long-duration centrifugal force. J Neurophysiol 39:970-984.

Fernández C, Goldberg JM (1976b) Physiology of peripheral neurons innervating otolith organs of the squirrel monkey. III. Response dynamics. J Neurophysiol 39:996-1008.

Fetsch CR, Wang S, Gu Y, Deangelis GC, Angelaki DE (2007) Spatial reference frames of visual, vestibular, and multimodal heading signals in the dorsal subdivision of the medial superior temporal area. J Neurosci 27:700-712.

Fushiki H, Barmack NH (1997) Topography and reciprocal activity of cerebellar Purkinje cells in the uvula-nodulus modulated by vestibular stimulation. J Neurophysiol 78:3083-3094.

Ghasia FF, Meng H, Angelaki DE (2008) Neural correlates of forward and inverse models for eye movements: evidence from three-dimensional kinematics. J Neurosci 28:5082-5087.

Gibson JJ (1950) The perception of visual surfaces. Am J Psychol 63:367-384.

Gilbert CD, Sigman M, Crist RE (2001) The neural basis of perceptual learning. Neuron 31:681-697.

Glasauer S (2003) Cerebellar contribution to saccades and gaze holding: a modeling approach. Ann N Y Acad Sci 1004:206-219.

Glasauer S, Schneider E, Grasso R, Ivanenko YP (2007) Space-time relativity in self-motion reproduction. J Neurophysiol 97:451-461.

Green AM, Angelaki DE (2003) Resolution of sensory ambiguities for gaze stabilization requires a second neural integrator. J Neurosci 23:9265-9275. 
Green AM, Angelaki DE (2004) An integrative neural network for detecting inertial motion and head orientation. J Neurophysiol 92:905-925.

Green AM, Shaikh AG, Angelaki DE (2005) Sensory vestibular contributions to constructing internal models of self-motion. J Neural Eng 2:S164-S179.

Green AM, Meng H, Angelaki DE (2007) A reevaluation of the inverse dynamic model for eye movements. J Neurosci 27:1346-1355.

Gu Y, Watkins PV, Angelaki DE, DeAngelis GC (2006) Visual and nonvisual contributions to three-dimensional heading selectivity in the medial superior temporal area. J Neurosci 26:73-85.

Gu Y, DeAngelis GC, Angelaki DE (2007) A functional link between area MSTd and heading perception based on vestibular signals. Nat Neurosci 10:1038-1047.

Gu Y, Angelaki DE, Deangelis GC (2008) Neural correlates of multisensory cue integration in macaque MSTd. Nat Neurosci 11:1201-1210.

Guldin WO, Akbarian S, Grüsser OJ (1992) Cortico-cortical connections and cytoarchitectonics of the primate vestibular cortex: a study in squirrel monkeys (Saimiri sciureus). J Comp Neurol 326:375-401.

Heuer HW, Britten KH (2004) Optic flow signals in extrastriate area MST: comparison of perceptual and neuronal sensitivity. J Neurophysiol 91:1314-1326.

Hwang EJ, Shadmehr R (2005) Internal models of limb dynamics and the encoding of limb state. J Neural Eng 2:S266-S278.

Ilg UJ (2008) The role of areas MT and MST in coding of visual motion underlying the execution of smooth pursuit. Vision Res 48:2062-2069.

Ito M (2005) Bases and implications of learning in the cerebellum-adaptive control and internal model mechanism. Prog Brain Res 148:95-109.

Jacobs RA (1999) Optimal integration of texture and motion cues to depth. Vision Res 39:3621-3629.

Kaas JH, Lyon DC (2007) Pulvinar contributions to the dorsal and ventral streams of visual processing in primates. Brain Res Rev 55:285-296.

Kawato M (1999) Internal models for motor control and trajectory planning. Curr Opin Neurobiol 9:718-727.

Kawato M, Wolpert D (1998) Internal models for motor control. Novartis Found Symp 218:291-304.

Kelly RM, Strick PL (2003) Cerebellar loops with motor cortex and prefrontal cortex of a nonhuman primate. J Neurosci 23:8432-8444.

Knill DC, Pouget A (2004) The Bayesian brain: the role of uncertainty in neural coding and computation. Trends Neurosci 27:712-719.

Lang W, Büttner-Ennever JA, Büttner U (1979) Vestibular projections to the monkey thalamus: an autoradiographic study. Brain Res 177:3-17.

Lisberger SG, Movshon JA (1999) Visual motion analysis for pursuit eye movements in area MT of macaque monkeys. J Neurosci 19:2224-2246.

Logan DJ, Duffy CJ (2006) Cortical area MSTd combines visual cues to represent 3-D self-movement. Cereb Cortex 16:1494-1507.

Marlinski V, McCrea RA (2009) Self-motion signals in vestibular nuclei neurons projecting to the thalamus in the alert squirrel monkey. J Neurophysiol 101:1730-1741.

Meng H, Angelaki DE (2008) Searching for vestibular and visual motion (optic flow) convergence in the macaque thalamus. Soc Neurosci Abstr 34:169.3.

Meng H, Bai RS, Sato H, Imagawa M, Sasaki M, Uchino Y (2001) Otolithactivated vestibulothalamic neurons in cats. Exp Brain Res 141:415-424.

Meng H, Green AM, Dickman JD, Angelaki DE (2005) Pursuit-vestibular interactions in brain stem neurons during rotation and translation. J Neurophysiol 93:3418-3433.

Meng H, May PJ, Dickman JD, Angelaki DE (2007) Vestibular signals in primate thalamus: properties and origins. J Neurosci 27:13590-13602.

Merfeld DM, Zupan LH (2002) Neural processing of gravitoinertial cues in humans. III. Modeling tilt and translation responses. J Neurophysiol 87:819-833.

Merfeld DM, Zupan L, Peterka RJ (1999) Humans use internal models to estimate gravity and linear acceleration. Nature 398:615-618.

Merfeld DM, Park S, Gianna-Poulin C, Black FO, Wood S (2005) Vestibular perception and action employ qualitatively different mechanisms. I. Frequency response of VOR and perceptual responses during Translation and Tilt. J Neurophysiol 94:186-198.

Middleton FA, Strick PL (2001) Cerebellar projections to the prefrontal cortex of the primate. J Neurosci 21:700-712.

Musallam S, Tomlinson RD (2002) Asymmetric integration recorded from vestibular-only cells in response to position transients. J Neurophysiol $88: 2104-2113$.
Page WK, Duffy CJ (1999) MST neuronal responses to heading direction during pursuit eye movements. J Neurophysiol 81:596-610.

Page WK, Duffy CJ (2003) Heading representation in MST: sensory interactions and population encoding. J Neurophysiol 89:1994-2013.

Rodman HR, Albright TD (1987) Coding of visual stimulus velocity in area MT of the macaque. Vision Res 27:2035-2048.

Royden CS, Banks MS, Crowell JA (1992) The perception of heading during eye movements. Nature 360:583-585.

Schor RH, Angelaki DE (1992) The algebra of neural response vectors. Ann N Y Acad Sci 656:190-204.

Shaikh AG, Ghasia FF, Dickman JD, Angelaki DE (2005a) Properties of cerebellar fastigial neurons during translation, rotation, and eye movements. J Neurophysiol 93:853-863.

Shaikh AG, Green AM, Ghasia FF, Newlands SD, Dickman JD, Angelaki DE (2005b) Sensory convergence solves a motion ambiguity problem. Curr Biol 15:1657-1662.

Shenoy KV, Bradley DC, Andersen RA (1999) Influence of gaze rotation on the visual response of primate MSTd neurons. J Neurophysiol 81:2764-2786.

Shidara M, Kawano K, Gomi H, Kawato M (1993) Inverse-dynamics model eye movement control by Purkinje cells in the cerebellum. Nature 365:50-52.

Shojaku H, Sato Y, Ikarashi K, Kawasaki T (1987) Topographical distribution of Purkinje cells in the uvula and the nodulus projecting to the vestibular nuclei in cats. Brain Res 416:100-112.

Si X, Angelaki DE, Dickman JD (1997) Response properties of pigeon otolith afferents to linear acceleration. Exp Brain Res 117:242-250.

Stocker AA, Simoncelli EP (2006) Noise characteristics and prior expectations in human visual speed perception. Nat Neurosci 9:578-585.

Strick PL, Dum RP, Fiez JA (2009) Cerebellum and non-motor function. Annu Rev Neurosci, in press.

Takahashi K, Gu Y, May PJ, Newlands SD, DeAngelis GC, Angelaki DE (2007) Multimodal coding of three-dimensional rotation and translation in area MSTd: comparison of visual and vestibular selectivity. J Neurosci 27:9742-9756.

Tanaka K, Hikosaka K, Saito H, Yukie M, Fukada Y, Iwai E (1986) Analysis of local and wide-field movements in the superior temporal visual areas of the macaque monkey. J Neurosci 6:134-144.

Tsodyks M, Gilbert C (2004) Neural networks and perceptual learning. Nature 431:775-781.

Upadhyay UD, Page WK, Duffy CJ (2000) MST responses to pursuit across optic flow with motion parallax. J Neurophysiol 84:818-826.

Voogd J, Gerrits NM, Ruigrok TJ (1996) Organization of the vestibulocerebellum. Ann N Y Acad Sci 781:553-579.

Warren WH (2003) Optic flow. In: The visual neurosciences (Chalupa LM, Werner JS, eds), pp 1247-1259. Cambridge, MA: MIT.

Warren WH Jr, Hannon DJ (1990) Eye movements and optical flow. J Opt Soc Am A 7:160-169.

Wolpert DM, Miall RC (1996) Forward models for physiological motor control. Neural Netw 9:1265-1279.

Wolpert DM, Ghahramani Z, Jordan MI (1995) An internal model for sensorimotor integration. Science 269:1880-1882.

Wylie DR, De Zeeuw CI, DiGiorgi PL, Simpson JI (1994) Projections of individual Purkinje cells of identified zones in the ventral nodulus to the vestibular and cerebellar nuclei in the rabbit. J Comp Neurol 349:448-463.

Yakusheva TA, Shaikh AG, Green AM, Blazquez PM, Dickman JD, Angelaki DE (2007) Purkinje cells in posterior cerebellar vermis encode motion in an inertial reference frame. Neuron 54:973-985.

Yakusheva TA, Blazquez PM, Angelaki DE (2008) Simple and complex spikes modulation of nodulus and uvula Purkinje cell during threedimensional translation and tilt. Soc Neurosci Abstr 34:18.9.

Yakushin SB, Raphan T, Cohen B (2006) Spatial properties of central vestibular neurons. J Neurophysiol 95:464-478.

Zupan LH, Merfeld DM, Darlot C (2002) Using sensory weighting to model the influence of canal, otolith and visual cues on spatial orientation and eye movements. Biol Cybern 86:209-230.

Zupan LH, Park S, Merfeld DM (2004) The nervous system uses internal models to achieve sensory integration. Conf Proc IEEE Eng Med Biol Soc 6:4487-4490. 